\title{
Ethical Issues in Neuroinformatics
}

Athanasios Alexiou, Georgia Theocharopoulou, and Panayiotis Vlamos

Department of Informatics, Ionian University, Plateia Tsirigoti 7, 49100 Corfu, Greece

\{alexiou, zeta. theo, vlamos\} @ionio.gr

\begin{abstract}
Scientific progress in Artificial Intelligence contributed to generate concepts of information processing and representation that provide a general sense of a unified theory of how the brain works. Comprehending the neurobiological basis of consciousness and memory mechanisms in the brain using information technology techniques involves mainly brain data analysis. Nowadays, there is an increasingly progress in the wide area of information technology applications to our understanding of neurological and psychological disorders. Neuroinformatics can enhance the development of systems that mimic brain storage and retrieval of information. In this paper we take into consideration the ethical implications that arise in the application of information-based methods in neuroscience research processes.
\end{abstract}

Keywords: Neuroinformatics, Artificial Intelligence, Bioethics.

\section{Introduction}

The exploration of brain functions from a variety of fields has led to scientific developments in Artificial Intelligence (AI), like computational methods and systems for learning and knowledge discovery. Neuroinformatics is a research field concerned with the study of neuroscience with the use of information technology. Neuroinformatics organizes and integrates neuroscience data and applies computational tools and mathematical models in order to understand human brain physiology and functionality (Fig 1). According to the International Neuroinformatics Coordinating Facility (INCF) there are three main fields where Neuroinformatics have been applied [1]:

- Development of tools and databases for management and sharing of neuroscience data at all levels of analysis

- Development of tools for analyzing and modelling neuroscience data

- Development of computational models of the nervous system and neural processes.

These areas of research are important for the integration and analysis of increasingly large-volume, high-dimensional, and fine-grain experimental data. Neuroinformaticians provide and create interoperable databases for clinical and research scientists. Therefore, it is necessary to be able to share data and findings. The field of Neuroinformatics came into existence when the Human Brain Project began in the early 1990's. The primary goal of the Human Brain Project and Neuroinformatics is the 
development of new technologies for creating databases and database search tools, for information exchange of neuroscience data, in order scientists and researchers to build data models of the brain and also create simulations of brain functions [2].

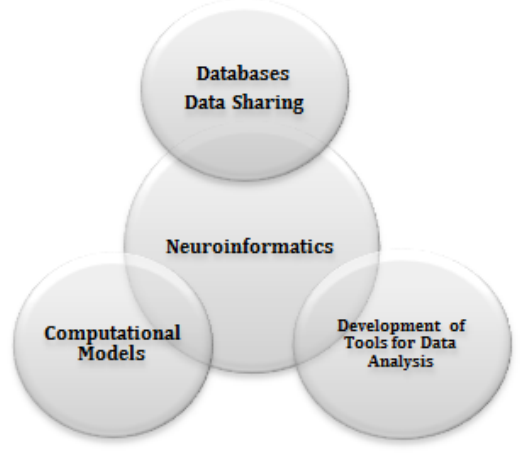

Fig. 1. Neuroinformatics Research Fields

Neuroinformatics has a variety of applications and uses in both the field of neuroscience and also information technology. Like other new technologies, there are legal and ethical issues that need to be accounted for in order to perform Neuroinformatics' research. There are several opinions on data sharing, and there are legal and ethical issues that need to be accounted for regarding four major key issues:

- Gathering data in order to create Neuroscience Databases

- Data sharing used in developing tools for analyses, simulation and modelling neuronal functions

- Data access in order to protect the subjects included in the data

- Legal and ethical issues that need to be accounted for performing research and clinical trials on patients.

Obviously, we have to distinguish the ethics of the productive technologies as a human's risk, from the ethics concerning the scientific and the AI's progress. The use of AI techniques in Neuroinformatics is totally separated from religious sentiments, human dignity or essential emotional states [3]. In this article we outline future directions for research regarding ethic challenges in the above mentioned issues.

\section{$2 \quad$ Legal and Ethical Issues in Neuroinformatics}

Data sharing projects include databases designed to archive data sets (e.g. neuroimaging results), and tools for neuroscience data. One major effort supporting integrative neuroscience research is the Neuroscience Information Framework (NIF). Few important aspects to the legal issues associated with Neuroinformatics regarding data and tool sharing are: protection for the creator of the data or tool, protection for the user and protection for the subjects included in the data [4]. 
Considering the availability and accessibility of data, it is very important for researchers and users to protect themselves and give credits to the appropriate researchers or institutions responsible for the research. But is this enough in order to achieve balance between the objective of scientific progress and the usage from sensitive groups of population? In which way will it become beneficial for all humans and more feasible to merge the increasing cost of new methods in health diagnosis and treatment, with the accessible and high quality medical care [5]?

It is also important for users to know the source of the information in order to be amenable to validation. In the past years there has been introduced national and international data-privacy regulations and Neuroinformatics researchers follow the copyright and intellectual property rights established by the Ethical Review Boards across the world [4]. While there are regulations and principles formulated with biomedical and social-behavioural research in mind, there are diverse opinions expressed by investigators about the fit between the national regulations and data sharing like: Should public money be spent on Open Access Projects, or how to share legally massive amounts of data?

In some cases further consideration is required; therefore several factors should be considered amongst others:

- proprietary information that must be protected

- legal and institutional requirements or regulations that need to be established

- collection of data, analysis of data, integration of the results into a conceptual model

- actual modelling of the experimental result, and development of a scientific manuscript

The above mentioned concepts should be considered in creating principles adequate for enabling collaborative and integrative information sharing in the field of neuroscience studies. The legal issues regarding sharing data have led to more strict and precise rules in order to ensure human subject anonymity and avoid inappropriate use of information. On the other hand international collaborations generated new concerns regarding fulfilment of International Ethical Review Boards requirements.

It is necessary that these cooperative efforts are carried out with similar and clearly defined fields, terminology description of data, models, experimental procedures etc., as well as developing appropriate common guidelines regarding legal issues of sharing and analyzing neuroscience data. Furthermore, credits should be appropriately addressed for both data owner, as well as data user. Some of the challenges regard also issues like the rights of each researcher the purpose of the data sharing, access to data, allocation of rights/ownership etc. Policy makers and developers should consider ethics and policy challenges regarding the following:

- Regulating the content of databases

- Access to databases

- Use of databases

The researchers should aim to ensure that data remains confidential and simultaneously develop models that fulfil their technical requirements in order to satisfy their expectations. 


\section{Regulating the Content of Databases}

Image-based data are primary drivers for Neuroinformatics efforts [6]. For example the functional Magnetic Resonance Imaging Data Center (fMRIDC) was established with the objective of creating a mechanism by which members of the Neuroscientific community might easily share functional neuroimaging data. Additionally, the Journal of Cognitive Neuroscience between 2000 and 2006 required that all authors who published in the journal submit their supplementary data to the fMRIDC [7]. Authors whose papers are based on results from datasets obtained from the Data Center are expected to provide descriptive meta-information for data use, credit original study authors and acknowledge the fMRIDC and accession number of the data set [8].

It is obvious that researchers using data from digital databases may encounter the risk of discovering violation of the providers' privacy, or disclosure of sensitive information. Since Neuroinformatics worldwide repositories will be populated by data, standardization of protocols for managing incidental findings, and limitation to the risk of intrinsic and consequential harm of donor are necessary. Ethical principles should enhance regulations for protecting human research participants, so as data sharing will always be safe and efficient.

Another issue that should be considered in neuroimaging sharing is the quality control of brain imaging data. Appropriate cautions should be applied in the use of these data, when predicting for example, diseases of the CNS. Concerns about the use of functional Magnetic Resonance Imaging (fMRI) scans of adolescents to predict later onset psychiatric disorders are referred in [9] regarding the reliability of neuroimaging due to the complexity and plasticity of the brain.

It is obvious that the hybrid ethical codes concern early diagnosis of CNS diseases differ from Neuroinformatics to traditional medicine approach due to the influence of other ethical frameworks and perspectives on their basic research and development. For some, this information could be helpful, empowering or enlightening and may enhance human health. For others, it is likely that such information could result in fear, anxiety and other mental health issues [3].

\section{$4 \quad$ Access and Use of Databases}

Artificial Intelligence research is undergoing a revolution in the area of understanding the mechanisms underlying intelligent behavior. Internet-accessibility of databases has enabled the usage of data from providers, patients, and research participants everywhere. Research studies involve a degree of trust between subjects and researchers, the former giving their consent and the latter protecting their privacy [10]. Different countries have various regulations and procedures regarding ethical issues of human data sharing:

- The federal human subject protection law in the United States mandates that all identifying information be removed from data prior to submission for sharing [11].

- Additionally, Institutional Review Boards (IRB) are administrative bodies in the U.S., established to protect the rights and welfare of human research subjects and are designated to review clinical investigations regulated by U.S. Food and Drug Administration (FDA). 
A major continuing goal of these boards is to ensure that national guidelines for protection of privacy and informed consent are best applied in the development of neuroscience data repositories. However, technical issues might arise, like the case where researchers wish to share retrospective data and process the way to obtain a new written informed consent. For example, the steering committee of the 1000 Functional Connectomes Project (FCP), a data sharing initiative project, realizing the necessity for privacy, agreed to a full anonymization of all datasets in accordance with the U.S. Health Insurance Portability and Accountability Act (HIPAA) [12].

Another aspect that raises further ethical issues is commercialization of databases. What details are necessary to share in data exchange and scientific results? The challenge is how to prevent exploitation of vulnerable populations, and avoid conflicts of interest. Data storing and data sharing can be both a challenge and a desire in for scientific researchers. The research of hybrid ethics in Neuroinformatics tools has to examine the malpractices during the past years in order to explore efficient and effective ways to accomplish data organization with ethical use of data. There is a worldwide debate about principles and regulations for the scientific processes. Guidance is needed to protect against discriminatory practices in case of commercialization of data sharing, as well as for issues regarding ownership and intellectual property of results and follow on innovation. A 'clear' definition on using and sharing data and meta-data seems to be necessary.

\section{Conclusion}

The next generation of neuroscientists contributes to a worldwide research network of excellence sharing data, tools, and information. Success on data sharing depends on the participation of the different types of neuroscience researchers. There should be considered a more critical thinking on searching for neuroscience relevant resources, including the ethical aspect. There is a major challenge in developing large-scale international collaborations and there is significant effort in developing integrated models of neuroscience processes. The more informed our brains are by science at all levels of analysis, the better will be our brains theoretical evolution [12]. Neuroinformatics can play a pivotal role in human brain research leading to innovations in neuroscience, informatics and treatment of brain disorders. Guidance to investigators, IRB representatives, public health officials and others regarding bioethics are key issues in neuroscience data sharing.

\section{References}

1. International Neuroinformatics Coordinating Facility, http: / /www. incf.org/documents/incf-core-documents / INCFStrategyOverview

2. Shepherd, G.M., Mirsky, J.S., Healy, M.D., Singer, M.S., Skoufos, E., Hines, M.S., Nadarni, P.M., Miller, P.L.: The Human Brain Project: Neuroinformatics tools for integrating, searching and modeling multidisciplinary neuroscience data. J. Neurophysiology 74 , 1810-1815 (1998) 
3. Alexiou, A., Psixa, M., Vlamos, P.: Ethical Issues of Artificial Biomedical Applications. In: Iliadis, L., Maglogiannis, I., Papadopoulos, H. (eds.) EANN/AIAI 2011, Part II. IFIP AICT, vol. 364, pp. 297-302. Springer, Heidelberg (2011)

4. Amari, S., Beltrame, F., Bjaalie, J.G., Dalkara, T., De Schutter, E., Egan, G.F., Goddard, N.H., Gonzalez, C., Grillner, S., Herz, A., Hoffmann, K.P., Jaaskelainen, I., Koslow, S.H., Lee, S.Y., Matthiessen, L., Miller, P.L., Da Silva, F.M., Novak, M., Ravindranath, V., Ritz, R., Ruotsalainen, U., Sebestra, V., Subramaniam, S., Tang, Y., Toga, A.W., Usui, S., Van Pelt, J., Verschure, P., Willshaw, D., Wrobel, A.: Neuroinformatics: the integration of shared databases and tools towards integrative neuroscience. Journal of Integrative Neuroscience 1(2), 117-128 (2002)

5. Alexiou, A., Vlamos, P.: Ethics at the Crossroads of Bioinformatics and Nanotechnology. In: 7th International Conference of Computer Ethics and Philosophical Enquiry (2009)

6. Martone, M.E., Amarnath, G., Ellisman, M.: E-neuroscience: challenges and triumphs in integrating distributed data from molecules to brains. Nature Neuroscience 7(5), 467-472 (2004)

7. Journal of Cognitive Neuroscience Instructions for Authors, J. Cognitive Neuroscience, http://jocn.mitpress.org/misc/ifora.shtml

8. Illes, J., Lombera, S.: Identifiable neuro ethics challenges to the banking of neuro data. Minn. JL Sci. \& Tech. 10, 71 (2008)

9. Fuchs, T.: Ethical issues in neuroscience. Current Opinion in Psychiatry 19(6), 600-607 (2006)

10. International Neuroinformatics Coordinating Facility, http: / / www. incf .org/documents / incf-coredocuments / INCFStrategyOverview

11. Wolf, L.E., Lo, B.: Untapped Potential: IRB Guidance for the Ethical Research Use of Stored Biological Materials. IRB: Ethics \& Human Res. 26(1), 1-8 (2004)

12. Maarten, M., Bharat, B.B., Castellanos, F.X., Milham, M.P.: Making data sharing work: The FCP/INDI experience. NeuroImage (2012), doi:10.1016/j.neuroimage.2012.10.064 